\title{
Gemeinsam für den Erhalt einer humanen Medizin
}

Im Jahr 2008 müssen wir uns gemeinsam mit verschiedenen politischen Fragen und - wahrscheinlich im nächsten Juni - mit einer äusserst wichtigen Abstimmung auseinandersetzen.

Wie Sie wissen, hat das Parlament vor den Festtagen den Entwurf für einen Verfassungsartikel («Art. 117a») verabschiedet, mit dem im Gesundheitswesen Konzepte eingeführt werden sollen, die mit der Medizin und den Arbeitsbedingungen, wie wir sie uns wünschen, schlicht und einfach unvereinbar sind. Diese Konzepte werden zwar mit schönen Aussagen und modernen Ideen auf ansprechende Weise verpackt und dargeboten, doch wir können es nicht hinnehmen, dass in unseren Praxen wirtschaftliche Überlegungen die Oberhand über menschliche Anliegen gewinnen!

Wir müssen uns jedoch bewusst sein, dass das, was für uns eine Selbstverständlichkeit ist, im Grunde bedeutet, dass für das Gesundheitssystem eine «Spezialregelung» verlangt wird. Diese entspricht nicht den Systemen, die in den anderen Lebensbereichen unseres Landes üblicherweise angewandt werden.

Es stellt sich somit die Frage, ob wir glaubwürdig sind, wenn wir uns für eine Medizin einsetzen, die nicht in erster Linie wirtschaftlichen, sondern menschlichen Aspekten Rechnung trägt.

Ja, wir können diesbezüglich unsere Anliegen durchaus glaubwürdig vertreten! Dies erfordert jedoch entsprechende Anstrengungen der gesamten Ärzteschaft, da man uns ausschliesslich nach unseren Taten beurteilen wird: Um glaubwürdig zu sein, müssen wir eine Politik betreiben, mit der unser medizinisches Engagement und unsere humanistischen Anliegen klar zum Ausdruck kommen. Ich meine damit, dass wir unsere Politik so betreiben müssen, wie wir als Ärztinnen und Ärzte tätig sind: mit einer einwandfreien Ethik, ohne Vorurteile und Ausschluss Andersdenkender sowie mit einer klaren Linie, die sich daran orientiert, was wir als gerecht beurteilen.

Dabei müssen wir ohne Komplexe agieren: Eine Politik, die sich nicht innerhalb der erwarteten Schemata bewegt, ist durchaus willkommen und wird auch verstanden. Und sie gibt uns die Möglichkeit, etwas anderes als nur die rigide Anwendung von Ideen zu verlangen, die gerade in Mode sind - mit einer solchen Politik können wir uns glaubwürdig für die Medizin einsetzen, die wir uns wünschen.

Als Beweis möchte ich auf die ausserordentlich hohe Teilnehmerzahl verweisen, die wir während der letzten Session an unserem Parlamentariertreffen verzeichnen konnten: Obwohl man nicht immer diesen Eindruck hat, ist die FMH in der Tat eine Institution mit politischem Einfluss!

Doch im Jahr 2008 kann man - weder wir noch unsere Partner/Gegner - nicht mehr Katz und Maus mit Umfragen, wohlwollenden Erklärungen und vermuteten Interessen der verschiedenen Beteiligten spielen: Die Stimmbürgerinnen und Stimmbürger können endlich selbst zur freien Arztwahl Stellung nehmen. Die Versicherer müssen die von ihnen unterzeichneten Verträge und die daraus resultierenden Tarifänderungen anwenden. Die Politiker müssen echte Entscheidungen zu den Patientenrechten und zum Datenschutz treffen, und die Verwaltung muss ihre Bereitschaft zur Zusammenarbeit unter Beweis stellen ...

Kurz gesagt, stehen in der Gesundheitspolitik einige Entscheide und Tatbeweise an, und die Reihen müssen geschlossen werden!

Selbstverständlich bereiten sich die FMH und alle ihre Abteilungen bereits aktiv auf diese neuen Herausforderungen in den Bereichen Politik, Recht, Tarifgestaltung usw. vor.

Was die politische Ebene anbelangt, werden die ersten Elemente, die für die Abstimmung vom Juni (oder September?) über den Verfassungsartikel zum Gesundheitssystem vorbereitet werden müssen, bereits an der Delegiertenversammlung Ende Januar vorgelegt. Da ein harter Abstimmungskampf bevorsteht, müssen wir uns mit möglichst vielen Mitteln darauf vorbereiten. Es geht dabei immerhin um unsere politische Präsenz und um unsere Arbeitsbedingungen in den nächsten Jahren.

Eine ethische Grundhaltung schliesst Kampfgeist nicht aus!

Dr. med. Jacques de Haller, Präsident der FMH 